\title{
Simulations on the Collisional Properties of Gas in Galaxies
}

\author{
Yves Revaz \\ Geneva Observatory, Switzerland \\ Daniel Pfenniger \\ Geneva Observatory \& University of Geneva, Switzerland
}

\begin{abstract}
In this work, we explore a simple method with few free parameters, which describes the global dynamical consequences on disk galaxies of a slow cycling of gas between the interstellar, almost collisionless very cold gas and the warm collisional phases, with a secular transformation of a fraction of warm gas into stars.
\end{abstract}

\section{The Model}

\subsection{Multiphase interstellar gas}

In our N-body model, particles have been divided into three distinct components: the stars (ST), the warm gas (WG) and the dark gas (DG). The warm gas represents interstellar gas with a large range of temperature $\left(10\right.$ to $\left.10^{5} \mathrm{~K}\right)$. It contains fragmented dense molecular clouds that are the location of star formation as well as gas at higher temperature heated by nearby star forming regions. The fractal structure of the ISM does not permit to assume it as a continuous collisional fluid. Standard methods like SPH are thus inappropriate and we have rather used the sticky particle scheme (Combes \& Gerin, 1985) that is better suited to simulate the clumpy collisional structures. The DG represents the very cold hydrogen $(T<10 \mathrm{~K})$ that is expected to exist in the outer disk of galaxies (Pfenniger et al. 1994). It represents a substantial fraction of the dark matter that behaves as collisonless. No further dark halo is used in this model.

\subsection{Mass and energy exchanges}

Mass and energy exchanges have been taken into account by introducing simple rules. Gaseous particles (WG and DG) can be heated by UV radiation of stars (including supernova feedback). WG particles are subject to radiative cooling. Once a DG particle is heated it can become WG. Likewise, a cooling WG particle can become DG. Thus all along the simulation a mass transfer between the gas phases occurs. Stars are created out of the WG component and are frozen in this state and the dynamical feedback of the star formation is also taken into account. 


\subsection{Initial conditions}

As initial conditions we have used a simple model based on a softened Mestel disk. The vertical profile is homogeneous between $\pm z_{\max }(R)$ where $z_{\max }(R)$ increases as a function of radius (flaring). All the matter is supposed to lie in the disk in the form of dark gas. In order to ignite star formation $5 \%$ of the initial mass is in the form of WG within $2 \mathrm{kpc}$.

\section{Results}

We have computed 11 models that explore the effect of the free parameters (heating, cooling, star formation, feedback) that leads to the following conclusions: soon after the beginning of the simulation, the galactic center is subject

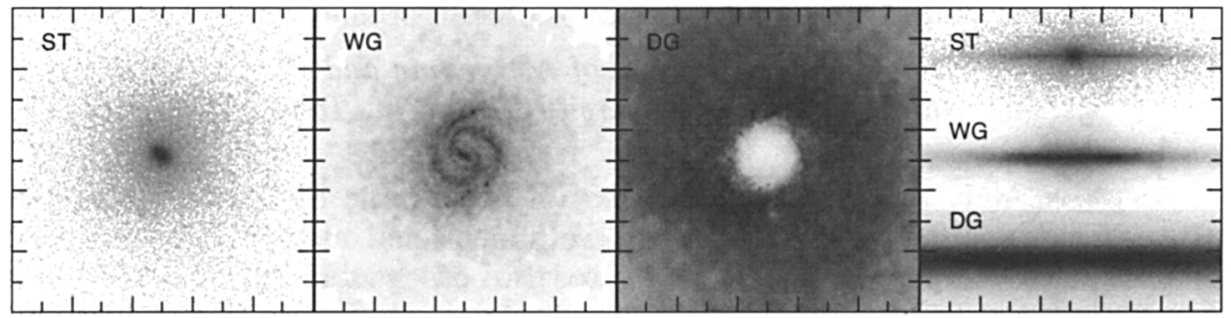

Figure 1. Projections of the reference model $(50 \times 50 \mathrm{kpc})$ at $t=$ $5900 \mathrm{Myr}$ ( $\mathrm{ST}=$ stars, $\mathrm{WG}=$ warm gas, $\mathrm{DG}=$ dark gas).

to an instability that results from the strong cooling. A bar forms and persists during the whole simulation. Spirals forms in the collisional component if the heating of the disk is not too high. They are usually two-armed but can also be flocculent if the rotation curve steeply decreases. Rings appear spontaneously at the OLR when the cooling of the warm gas is sufficient. They are formed by orbits captured by $2: 1$ resonances. Inner ILR rings are also observed. An exponential visible disk as well as a bulge emerge out of the initial Mestel disk. The bulge is more prominent for evolved models. Due to the star heating, the dark gas distribution is depleted at the center of the galaxy. It is anti-correlated with stars. The warm gas distribution decreases at the center. Its mass fraction ranges between 0.02 and 0.12 . The ratio of dark over visible matter increases as a function of time.

Thus, the extreme assumption of a heavy disk containing all the matter including the dark matter is not in contradiction with observational facts. On the contrary, it reproduces naturally common observed features of spiral galaxies as well as the evolution of galaxies all along the Hubble sequence from Sd to Sa. A full discution of all simulations will apear in a forthcoming paper.

\section{References}

Combes F., Gerin M., 1985, A\&A, 150, 327

Pfenniger D., Combes F., Martinet L., 1994, A\&A, 285, 79 